\title{
TRANSFORMATIONS THAT DO NOT ACCEPT A FINITE INVARIANT MEASURE ${ }^{1}$
}

\author{
BY ARSHAG HAJIAN AND YUJI ITO
}

In the following we shall consider only nonatomic, $\sigma$-finite measure spaces $(X, \mathfrak{B}, m)$. We say that a measure $\mu$ is equivalent to $m$ if $\mu$ and $m$ have the same sets of measure zero. We shall discuss measurable transformations $T$ that are 1-1 onto maps with measurable inverses. If $m(T A)=m(A)$ for all sets $A \in \mathscr{B}$ we say that $T$ is a measure preserving transformation, or that $m$ is an invariant measure for the transformation $T$. We assume that all transformations mentioned are nonsingular; in other words, the image of a set of positive measure has positive measure also. A measurable transformation $T$ is ergodic if $T A=A$ implies that either the set $A$ or its complement has measure zero. We shall often tacitly assume the phrase "almost everywhere", and all sets considered shall be measurable.

In [13] E. Hopf first discussed a necessary and sufficient condition for the existence of a finite invariant measure $\mu$ equivalent to $m$. Since then many authors have discussed different aspects of transformations without a finite invariant measure and have obtained a number of interesting results that have deep connections with other areas of mathematics. For instance, there is a significant influence on the classification theory of the factors of a von Neumann algebra.

In [10] weakly wandering sets for a transformation $T$ were introduced, and it was shown that there exists a finite measure $\mu$ equivalent to $m$ and invariant for $T$ if and only if there are no weakly wandering sets of positive measure for the transformation $T$. Let $\mathscr{W}=\left\{n_{i} \mid i=0,1,2, \ldots\right\}$ be a sequence of integers; we say that a set $A$ is a weakly wandering set under the sequence $ण$ for the transformation $T$ if the sets $T^{n_{i}} A$ for $i=0,1,2, \ldots$ are mutually disjoint. If the weakly wandering set $A$ has positive measure then we say that $\mathscr{W}$ is a weakly wandering sequence for the transformation $T$. In case the sequence $\mathcal{W}$ consists of the set of all integers then we have the familiar case where $A$ is a wandering set.

Recurrent transformations play an important role in ergodic theory; these are the transformations which do not accept wandering sets of positive measure. Strongly recurrent transformations were introduced and discussed in [3]; these are the transformations which do not accept weakly wandering sets of positive measure. An ergodic measure preserving transformation $T$ is

This is an expanded version of an invited address presented by the first author at the 82nd annual meeting of the Society in San Antonio, Texas, on January 22, 1976; received by the editors September 28, 1977.

AMS (MOS) subject classifications (1970). Primary 28A65, 28A70, 43A05.

'Work supported in part by grants from the NSF.

๑) American Mathematical Society 1978 
also a recurrent transformation; moreover, if it is defined on an infinite measure space $(X, \mathfrak{B}, m)$ then it cannot accept a finite invariant measure $\mu$ equivalent to $m$. It is clear then that the strongly recurrent transformations are the ones identified with the class of transformations that accept a finite invariant measure $\mu$ equivalent to $m$. In this article we concentrate on the weakly wandering sequences of a measurable transformation $T$. These and other related sequences exist when $T$ does not possess a finite invariant measure.

In the past several years while working on different problems of ergodic theory we have obtained several results concerning weakly wandering sequences. On numerous occasions we have collaborated closely with $\mathbf{S}$. Kakutani, and many of the results we discuss here and elsewhere have been a product of our joint efforts. In this article we shall not attempt to give a comprehensive treatment of the theory of transformations without a finite invariant measure. Rather, we shall restrict our attention to properties of weakly wandering and other related sequences associated with such transformations. We shall present proofs and examples only when they supplement the existing literature. We shall cite only those works from the literature that have an immediate connection with our treatment.

The weakly wandering sequences of an ergodic measure preserving transformation $T$ defined on an infinite measure space reflect strongly the behaviour of $T$. One example of this is exhibited in Theorem 1 which was discussed briefly in [14].

THEOREM 1. Let $T$ be an ergodic measure preserving transformation defined on the infinite measure space $(X, \mathscr{B}, m)$, and let $\mathscr{W}=\left\{n_{i} \mid i=0,1,2, \ldots\right\}$ be a weakly wandering sequence for the transformation $T$. Then for any set $A$ with $0<m(A)<\infty$ we have

$$
\begin{array}{r}
\lim _{k \rightarrow \infty} m\left(\bigcup_{i=0}^{k-1} T^{i} A\right)=\infty, \\
\lim _{k \rightarrow \infty} \frac{1}{k} m\left(\bigcup_{i=0}^{k-1} T^{i} A\right)=0, \\
\lim _{k \rightarrow \infty} \frac{1}{k} m\left(\bigcup_{i=0}^{k-1} T^{n_{i} A}\right)>0 .
\end{array}
$$

Proof. We let

$$
A^{*}=\bigcup_{i=0}^{\infty} T^{i} A .
$$

Since the set $B=A^{*}-T A^{*}$ is a wandering set for the transformation $T$, and $T$ is ergodic we have $T A^{*}=A^{*}$. Then (a) is an immediate consequence of the fact that $m(X)=\infty$.

To show (b) we let

$$
B_{n}=\bigcup_{i=0}^{n} T^{i} A \text { for } n=0,1,2, \ldots,
$$


$C_{0}=B_{0}$, and $C_{n}=B_{n}-B_{n-1}$ for $n=1,2, \ldots$ It follows that

$$
A^{*}=\bigcup_{i=0}^{\infty} T^{i} A=\bigcup_{n=0}^{\infty} B_{n}=\bigcup_{n=0}^{\infty} C_{n} \text { (disj), }
$$

$\left\{T^{-n} C_{n} \mid n=0,1,2, \ldots\right\}$ is a monotone decreasing sequence of sets of positive measure contained in $A$, and

$$
C=\bigcap_{n=0}^{\infty} T^{-n} C_{n}
$$

is a wandering set for $T$. Therefore, $m(C)=\lim _{n \rightarrow \infty} m\left(T^{-n} C_{n}\right)=0$ which implies that $\lim _{n \rightarrow \infty} m\left(C_{n}\right)=0$. Since the sets $C_{n}$ for $n=0,1,2, \ldots$ are mutually disjoint, it follows that

$$
\begin{aligned}
0 & =\lim _{k \rightarrow \infty} \frac{1}{k} \sum_{n=0}^{k-1} m\left(C_{n}\right)=\lim _{k \rightarrow \infty} \frac{1}{k} m\left(\bigcup_{n=0}^{k-1} C_{n}\right) \\
& =\lim _{k \rightarrow \infty} \frac{1}{k} m\left(\bigcup_{n=0}^{k-1} B_{n}\right)=\lim _{k \rightarrow \infty} \frac{1}{k} m\left(\bigcup_{n=0}^{k-1} T^{n} A\right) .
\end{aligned}
$$

To show (c) we let $D$ be a weakly wandering set of positive measure under the sequence $\mathcal{W}=\left\{n_{i} \mid i=0,1,2, \ldots\right\}$ for the transformation $T$ and let $A$ be any set of positive measure. Since $T$ is ergodic it follows that there exists a positive integer $p>0$ such that the set $B=T^{p} D \cap A$ has positive measure. Since $B \subset T^{p} D$ it follows that $B$ is also a weakly wandering set under the same sequence $\mathcal{W}$ for the transformation $T$. For $k=1,2, \ldots$ we then have

$$
m\left(\bigcup_{i=0}^{k-1} T^{n_{i}} A\right)>m\left(\bigcup_{i=0}^{k-1} T^{n_{i}} B\right)=\sum_{i=0}^{k-1} m\left(T^{n_{i}} B\right)=k \cdot m(B) .
$$

Dividing the above expression by $k$ we obtain

$$
\frac{1}{k} m\left(\bigcup_{i=0}^{k-1} T^{n_{i}} A\right)>m(B)>0,
$$

and this completes the proof of the theorem.

We observe from Theorem 1 that when $T$ is an ergodic measure preserving transformation defined on an infinite measure space then the proof of (a) says that the union of the successive images under the transformation $T$ of any set $A$ of positive measure grows in size to fill up the space $X$. From (b) on the other hand, we see that this growth cannot be too fast and must be slow enough to satisfy the ergodicity of $T$. However, (c) shows that there exists a lower bound to the rate of this growth which is independent of the set $A$ of positive measure but is related to the "thickness" of the weakly wandering sequences of $T$. Since the above properties are preserved under spatial isomorphism of transformations, it is possible to make these general observations more specific. As mentioned in [14] employing these ideas one can construct examples of ergodic measure preserving transformations defined on an infinite measure space which are not isomorphic to each other.

In another development [1] similar ideas have been considered and applied 
successfully in the study of random walks on the integers. By slightly refining the definition of a weakly wandering sequence one obtains the interesting notion of an eventually weakly wandering sequence; namely, let $T$ be a measurable transformation defined on the measure space $(X, \Re, m)$, and assume that $m(X)<\infty$. Then $\left\{n_{i} \mid i=0,1,2, \ldots\right\}$ is an eventually weakly wandering sequence for the transformation $T$ if for any set $A$ of positive measure and every $\varepsilon>0$ there exists a positive integer $p>0$ and a set $A^{\prime} \subset A$ with $m\left(A^{\prime}\right)<\varepsilon$ such that the set $B=A-A^{\prime}$ is a weakly wandering set under the sequence $\left\{n_{i} \mid i \geqslant p\right\}$ for $T$. In [1] eventually weakly wandering sequences were introduced and studied in some detail. In [2] examples of such sequences were constructed, and it was shown that they possess a number of novel properties.

As noted above, the "density" of a weakly wandering sequence influences the rate of growth of the union of the successive images of a set $A$ of positive measure under the iterates of the transformation $T$. It is clear that if $T$ is an ergodic measure preserving transformation defined on an infinite measure space then $T$ admits weakly wandering sequences but not wandering sequences; stating it differently, a weakly wandering sequence for an ergodic transformation must be "sparse". In fact, it can be seen from the literature [6] and from Theorem 1 that the density of a weakly wandering sequence $\left\{n_{i} \mid i=0,1,2, \ldots\right\}$ for an ergodic measure preserving transformation defined on an infinite measure space must be zero; or $\lim _{i \rightarrow \infty} i / n_{i}=0$. However, a more general result is true.

THEOREM 2. Let $T$ be a recurrent transformation defined on the measure space $(X, \Re, m)$, and let $\mathscr{W}=\left\{n_{i} \mid i=0,1,2, \ldots\right\}$ be a weakly wandering sequence for $T$. Then there exists an increasing sequence of positive integers $\mathcal{V}=\left\{k_{i} \mid i=\right.$ $0,1,2, \ldots\}$ such that the sequence $\mathscr{W}+\mathcal{V}=\left\{n_{i}+k_{j} \mid i=0,1,2, \ldots, j=\right.$ $0,1,2, \ldots\}$ is a direct sum of the sequences $\mathscr{W}$ and $\mathfrak{V}$ in the following sense: if $n+k=n^{\prime}+k^{\prime}$ for $n, n^{\prime} \in \mathcal{W}$, and $k, k^{\prime} \in \mathcal{V}$ then $n=n^{\prime}$ and $k=k^{\prime}$.

In order to prove Theorem 2 we first prove the following lemma:

LEMMA. Let $T$ be a recurrent transformation defined on the measure space $(X, \mathfrak{B}, m)$, and let $\mathscr{W}=\left\{n_{i} \mid i=0,1,2, \ldots\right\}$ be a weakly wandering sequence for $T$. Then there exists a positive integer $k>0$ such that $\{\mathscr{W}+k\} \cap \mathcal{W}=$ $\varnothing$ and $\{\mathscr{W}+k\} \cup$ W is again a weakly wandering sequence for $T$, where $\{$ ข $+k\}=\left\{n_{i}+k \mid i=0,1,2, \ldots\right\}$.

Proof. Let $A$ be a set of positive measure and weakly wandering under the sequence $\mho=\left\{n_{i} \mid i=0,1,2, \ldots\right\}$ for $T$. Since $T$ is a recurrent transformation there exists an integer $k>0$ such that the set $T^{k} A \cap A$ has positive measure. We let $B=T^{-k} A \cap A$ and $C=B-T^{k} B$. Since $B$ is contained in $A$ it is also a weakly wandering set, and therefore $m(C)>0$. It follows then that $\{\mathscr{W}+k\} \cap \mathscr{W}=\varnothing$, and $C$ is a set of positive measure which is weakly wandering under the sequence $\{\partial+k\} \cup \mho$. This completes the proof of the lemma.

ProOF OF TheOREM 2. Let $\mathscr{W}=\left\{n_{i} \mid i=0,1,2, \ldots\right\}$ be a weakly wandering sequence for the transformation $T$, and let $\mathscr{W}_{0}=W$. By the lemma we obtain a positive integer $k_{0}>0$ such that $\left\{\mho_{0}+k_{0}\right\} \cap \mho_{0}=\varnothing$ and $\mho_{1}=$ 
$\left\{\mathscr{w}_{0}+k_{0}\right\} \cup \mathcal{W}_{0}$ is a weakly wandering sequence for $T$. We note that the integer $k>0$ specified in the lemma can be chosen arbitrarily large. Therefore, using induction we choose an increasing sequence of positive integers $\mathfrak{V}=\left\{k_{i} \mid i=1,2, \ldots\right\}$, and for each $i=1,2, \ldots$ we define the sequence $\mho_{i}=\left\{\mathscr{w}_{i-1}+k_{i-1}\right\} \cup \mho_{i-1}$ such that $\left\{\mho_{i}+k_{i}\right\} \cap \mho_{i}=\varnothing$ and $\mathscr{W}_{i+1}=$ $\left\{\mathscr{w}_{i}+k_{i}\right\} \cup \mathscr{W}_{i}$ is a weakly wandering sequence for $T$. Finally, we let $n$, $n^{\prime} \in \mathcal{W}$ and $k, k^{\prime} \in \mathcal{T}$. We assume that $k<k^{\prime}$ and $k^{\prime}=k_{p}$ for some positive integer $p>0$. We then have $n+k \in \mathcal{W}_{p}$ and $n^{\prime}+k^{\prime} \in\left\{\mathcal{W}_{p}+k_{p}\right\}$, and this completes the proof of the theorem.

An interesting property of weakly wandering sequences of an ergodic measure preserving transformation $T$ is reflected in the frequency of visits to a set $A$ of the images of a point $x$ under the iterates of $T$. In particular, it is shown in [5] that if $\left\{n_{i} \mid i=0,1,2, \ldots\right\}$ is a weakly wandering sequence for the ergodic measure preserving transformation $T$ defined on the infinite measure space $(X, \Re, m)$ and $A$ is any set of finite measure, then the suborbit $\left\{T^{n_{i}} x \mid i=0,1,2, \ldots\right\}$ of almost all points $x \in X$ intersects the set $A$ at only finitely many points. We call sequences with such a property dissipative. More generally, we have the following definition.

DEFINITION 1. Let $T$ be a measure preserving transformation defined on the measure space $(X, \Re, m)$. A sequence of integers $\left\{n_{i} \mid i=0,1,2, \ldots\right\}$ is said to be a dissipative sequence for the transformation $T$ if for any integrable function $f \in L_{1}(X, \Re, m)$ we have

$$
\sum_{i=0}^{\infty}\left|f\left(T^{n_{i}} x\right)\right|<\infty \quad \text { almost everywhere. }
$$

It is not difficult to show that a weakly wandering sequence $\left\{n_{i} \mid i=0,1\right.$, $2, \ldots\}$ is also a dissipative sequence for an ergodic measure preserving transformation defined on an infinite measure space; see [6]. The converse however, is not true. We show this with an example that is constructed by slightly modifying the example in [11].

EXAMPLE. We consider the one sided infinite direct product measure space of two point spaces; namely, $\left(X_{0}, \mathscr{B}_{0}, m\right)=\prod_{i=0}^{\infty}\left(Y_{i}, \mathscr{F}_{i}, m_{i}\right)$ where $Y_{i}=$ $\{0,1\}, \mathscr{F}_{i}$ consists of all subsets of $Y_{i}$, and $m_{i}(0)=m_{i}(1)=1 / 2$ for $i=0,1$, $2, \ldots$ We also assume that we have removed from the measure space $\left(X_{0}, \mathscr{B}_{0}, m\right)$ all the dyadic rationals; namely, the countable set consisting of all points $x=\left(y_{0}, y_{1}, \ldots\right) \in X_{0}$ with the property that $y_{i} \in Y_{i}$ are constant for all $i \geqslant p$, where $p=p(x)$ is a nonnegative integer. We consider the rectangular sets

$$
A_{n}=\left\{x \in X_{0} \mid x=\left(1, \ldots, \ldots, 1,0, y_{n+1}, \ldots\right)\right\}
$$

for $n=0,1,2, \ldots$, and defined $T_{0} x=\left(0, \ldots, 0,1, y_{n+1}, \ldots\right)$ for $x \in A_{n}$, $n=0,1,2, \ldots$ It is clear then that $T_{0}$ is an ergodic measure preserving transformation defined on the measure space $\left(X_{0}, \mathscr{B}_{0}, m\right)$ with $m\left(X_{0}\right)=1$. We note that $T_{0}$ is also what is referred to in the literature [9] as a von Neumann transformation. We now construct a measure space $(X, \mathscr{B}, m)$ and a transformation $T$ which is built up from the transformation $T_{0}$ on top of the set $X_{0}$ via the skyscraper or stacking method; see [8]. We let $f(0)=0$ and 
suppose that an increasing sequence of positive integers $\{f(n) \mid n=1,2, \ldots\}$ has been defined (we shall soon define such a sequence inductively below). For $n=0,1,2, \ldots$ we let $\left(A_{n}, 0\right)=A_{n}$ as defined in (1) and let $\left(A_{n}, i\right)$ be an isomorphic copy of $A_{n}$ for $0<i \leqslant f(n)$. We denote by the same letter $S$ all the isomorphisms:

$$
S\left(A_{n}, i\right)=\left(A_{n}, i+1\right) \text { for } i=0,1, \ldots, f(n)-1 \text {, and } n=1,2, \ldots
$$

We next consider the measure space $(X, \mathfrak{B}, m)$, where

$$
X=\bigcup_{n=0}^{\infty} \bigcup_{i=0}^{f(n)}\left(A_{n}, i\right) \quad(\mathrm{disj})
$$

$\mathscr{B}$ is the extension to the space $X$ of the $\sigma$-field $\mathscr{B}_{0}$ of the "Lebesgue measurable" subsets of $X_{0}$, and $m$ is the corresponding extension to the measure space $(X, \mathfrak{B})$ of the measure $m$ defined on $\left(X_{0}, \mathscr{B}_{0}\right)$; see also [11] for a similar construction. Finally, we define the transformation $T$ on the measure space $(X, \mathscr{B}, m)$ by:

$$
T x=\left\{\begin{array}{l}
S x \text { if } x \in\left(A_{n}, i\right) \text { for } i=0,1, \ldots, f(n)-1, \text { and } n=1,2, \ldots, \\
T_{0} S^{-f(n)} x \text { if } x \in\left(A_{n}, f(n)\right) \text { for } n=0,1,2, \ldots
\end{array}\right.
$$

It is clear then that $T$ is an ergodic measure preserving transformation defined on the measure space $(X, \mathfrak{B}, m)$.

We now choose the increasing sequence of positive integers $\{f(n) \mid n=1$, $2, \ldots\}$ mentioned above, and at the same time choose another sequence $\mathscr{W}=\left\{n_{i} \mid i=0,1,2, \ldots\right\}$ of positive integers inductively as follows: Let us consider the sets

$$
B_{k}=\bigcup_{n=k}^{\infty} \bigcup_{i=f(k-1)+1}^{f(k)}\left(A_{n}, i\right) \text { for } k=1,2, \ldots
$$

We let $f(0)=0$ and $n_{0}=0$. Looking carefully at the images of the set $X_{0}$ by the transformation $T$ it is clear that if the integer $f(1)>0$ is chosen large enough, then there exists some other positive integer $n_{1}>0$ such that the sets $T^{n_{1}} X_{0}$ and $T^{n_{1}+1} X_{0}$ are both contained in the set $B_{1}$. Therefore, we choose the integers $f(1)>0$ and $n_{1}>0$ with that property in mind. Next we suppose that the integers $0=f(0)<f(1)<\cdots<f(k)$ and $0=n_{0}<n_{1}<\cdots<$ $n_{k}$ have been chosen with the property that for $i=1,2, \ldots, k$

$$
T^{n_{i}} X_{0} \cup T^{n_{i}+i} X_{0} \subset B_{i} \text {. }
$$

Then it is similarly clear that we can choose the integer $f(k+1)>f(k)$ large enough such that for some other positive integer $n_{k+1}>n_{k}(2)$ is satisfied for $i=k+1$ also. In this way we are able to define inductively the sequence of integers $\{f(n) \mid n=0,1,2, \ldots\}$ and simultaneously choose another sequence of positive integers $\left\{n_{i} \mid i=0,1,2, \ldots\right\}$ such that (2) is satisfied for all $i=1$, $2, \ldots$. Since the sets $B_{n}$ for $n=1,2, \ldots$ and the set $X_{0}$ are all mutually disjoint, from (2) follows that $X_{0}$ is a weakly wandering set under the 
sequence $\mho=\left\{n_{i} \mid i=0,1,2, \ldots\right\}$ and also under the sequence $\mho^{\prime}=\left\{n_{i}\right.$ $+i \mid i=0,1,2, \ldots\}$ for $T$.

It is clear from the above example that the sequence $\mathfrak{V}=\mathscr{W} \cup \mathcal{W}$ is a dissipative sequence for the transformation $T$ since $\mathcal{V}$ is the union of two of its weakly wandering sequences. However, the sequence $\mathcal{V}$ is not a weakly wandering sequence for $T$, in fact, $\mathfrak{T}$ cannot be a weakly wandering sequence for any recurrent transformation. This is a consequence of the following observation:

Suppose $A$ is a set of positive measure which is weakly wandering under the sequence $\mathcal{V}$ for a recurrent transformation $T$. Then for any two integers $p$, $q \in \mathfrak{V}, p \neq q, T^{p} A \cap T^{q} A=\varnothing$ implies that $T^{p-q} A \cap A=\varnothing$. Also from the construction of the sequence $\mathfrak{V}=\mathscr{W} \cup \mathcal{W}$ it is clear that $\mathfrak{V}-\mathfrak{V}=\{p-$ $q \mid p, q \in \mathfrak{V}\}$ consists of the set of all integers. These two facts imply that the set $A$ is in fact a wandering set of positive measure for $T$, and this is a contradiction to $T$ being recurrent.

In view of the above example it is clear that there exist dissipative sequences which are not weakly wandering sequences for an ergodic measure preserving transformation. However, we do not know of any other method by which we can construct a dissipative sequence for an ergodic measure preserving transformation defined on an infinite measure space other than by taking the union of a finite number of its weakly wandering sequences. In fact, in this direction we are not able to show whether a dissipative sequence for such a transformation must be of density zero or not.

Closely related to the weakly wandering and dissipative sequences of an ergodic measure preserving transformation $T$ defined on an infinite measure space are the recurrent sequences of $T$. These sequences were first introduced and discussed in some detail in [6]. A sequence $\Re=\left\{r_{i} \mid i=0,1,2, \ldots\right\}$ is a recurrent sequence for a transformation $T$ if the intersection of the sequence $\Re$ with any weakly wandering sequence $\mathcal{W}$ of $T$ consists of a finite number of integers. Unlike weakly wandering or dissipative sequences however, recurrent sequences do not always exist for ergodic measure preserving transformations defined on an infinite measure space. For example, ergodic measure preserving transformations that are of type zero do not accept recurrent sequences, while those that are of type positive always have them. The type of an ergodic measure preserving transformation was first discussed in [10]. For a real number $\alpha$ with $0 \leqslant \alpha \leqslant 1$ a transformation $T$ is of type $\alpha$ if $\lim \sup _{n \rightarrow \infty} m\left(T^{n} A \cap A\right)=\alpha m(A)$ for all sets $A$ with $0<m(A)<\infty$; and more generally, a transformation $T$ is of type positive if $\lim _{\sup } \rightarrow \infty m\left(T^{n} A\right.$ $\cap A)>0$ for all sets $A$ of positive measure. In [12] examples of ergodic measure preserving transformations are constructed on an infinite measure space which contain transformations of type $\alpha$ for every $\alpha$ with $0 \leqslant \alpha \leqslant 1$. We refer to [17] also for a discussion of ergodic transformations of type zero or type positive. Recurrent sequences are shown to exist for the ergodic measure preserving transformation $T$ constructed on the infinite measure space of the real line in [11], where a complete description and a listing of all the recurrent sequences of that example are described.

Let us suppose that $T$ is an ergodic measure preserving transformation defined on a measure space $(X, \mathfrak{B}, m)$. From the fact that any measurable 
function which is invariant under $T$ has to be a constant almost everywhere follows that if two measures are equivalent and invariant under $T$ then one must be a constant multiple of the other. From this it is easy to conclude that if a measurable transformation $S$ commutes with $T$ then there exists a constant $K>0$ such that $m(S A)=K m(A)$ for all $A \in \mathscr{B}$. In case $(X, \mathscr{B}, m)$ is a finite measure space then the situation simplifies slightly, and any measurable transformation $S$ which commutes with an ergodic measure preserving transformation $T$ defined on a finite measure space $(X, \Re, m)$ preserves the same measure $m$ also. In case $m(X)=\infty$ however, the situation is more complicated. The transformation constructed in [11] is an example of an ergodic measure preserving transformation $T$ defined on the infinite measure space $(X, \mathscr{B}, m)$ of the real line. Among other properties of the transformation $T$ it is shown that if $S$ is any measurable transformation defined on the same measure space $(X, \mathscr{B}, m)$ which commutes with $T$, then $S$ must preserve the same measure $m$ also. The crucial property that $T$ possesses in the above example happens to be reflected in some special weakly wandering sequences. In order to clarify these ideas and show their effect on some related results we introduce the following definitions:

Definition 2. A weakly wandering sequence $\mho \widehat{C}=\left\{n_{i} \mid i=0,1,2, \ldots\right\}$ for the measurable transformation $T$ defined on the measure space $(X, \Re, m)$ is said to be an exhaustive weakly wandering sequence for $T$ if

$$
X=\bigcup_{i=0}^{\infty} T^{n_{i} B} \quad \text { (disj) }
$$

for some set $B \in \mathscr{B}$. In this case we also say that $B$ is an exhaustive weakly wandering set under the sequence $\mho$ for $T$.

Definition 3. A weakly wandering sequence $\mathscr{W}=\left\{n_{i} \mid i=0,1,2, \ldots\right\}$ for the measure preserving transformation $T$ defined on the infinite measure space $(X, \mathfrak{B}, m)$ is a weakly wandering sequence of infinite type for $T$ if there exists a set $A$ of infinite measure which is weakly wandering under the sequence $W$. In case the weakly wandering sequence $\mathscr{W}$ is not of infinite type then we say that it is a weakly wandering sequence of finite type. Finally, we say that the weakly wandering sequence $\mathcal{W}$ is of bounded type if there exists a constant $K>0$ such that if $A$ is any weakly wandering set under the sequence $\mho$ then $m(A) \leqslant K$.

There are two results in the literature that are quite general in nature and relate directly to these definitions. In [4] it is shown that every ergodic measure preserving transformation defined on an infinite measure space possesses weakly wandering sequences of infinite type. In [16] it is shown that every ergodic transformation $T$ defined on a measure space $(X, \mathfrak{B}, m)$ which does not admit a finite invariant measure $\mu$ equivalent to $m$ possesses exhaustive weakly wandering sequence. However, the example of the ergodic measure preserving transformation $T$ constructed in [11] is more restrictive. The example possesses some special properties that are not shared generally by ergodic measure preserving transformations defined on an infinite measure space. The following theorem explains one such property.

THEOREM 3. Let $T$ be an ergodic measure preserving transformation defined 
on the infinite measure space $(X, \Re, m)$. Let $B$ be a set of finite measure which is an exhaustive weakly wandering set under the sequence $\mho \sigma=\left\{n_{i} \mid i=0,1\right.$, $2, \ldots\}$ for $T$. Then W is a weakly wandering sequence of bounded type for $T$; moreover, any measurable transformation $S$ which commutes with $T$ must preserve the same measure $m$ also.

In order to prove Theorem 3 we first prove the following lemma:

LEMMA. Let $T$ be a measure preserving transformation defined on the measure space $(X, \mathscr{B}, m)$. Let $B$ and $C$ be two weakly wandering sets under the same sequence $\mho \mathrm{S}=\left\{n_{i} \mid i=0,1,2, \ldots\right\}$ for $T$, and assume that

$$
C \subset \bigcup_{i=0}^{\infty} T^{n_{i}} B(\mathrm{disj})
$$

Then $m(C) \leqslant m(B)$.

Proof. From the hypotheses follows that

$$
C=\bigcup_{i=0}^{\infty} T^{n_{i}} B \cap C \quad \text { (disj). }
$$

Since $T$ is a measure preserving transformation we have

$$
m(C)=\sum_{i=0}^{\infty} m\left(T^{n_{i}} B \cap C\right)=\sum_{i=0}^{\infty} m\left(B \cap T^{-n_{i}} C\right) .
$$

It is clear that the set $C$ is also weakly wandering under the sequence $W^{\prime}=\left\{-n_{i} \mid i=0,1,2, \ldots\right\}$; therefore,

$$
\sum_{i=0}^{\infty} m\left(B \cap T^{-n_{i}} C\right)=m\left(\bigcup_{i=0}^{\infty} B \cap T^{-n_{i}} C\right) \leqslant m(B) .
$$

Combining (3) and (4) we conclude the proof of the lemma.

Proof of Theorem 3. Suppose there exists a set $B$ with $m(B)<\infty$ such that

$$
X=\bigcup_{i=1}^{\infty} T^{n_{i} B} \quad \text { (disj) }
$$

for the weakly wandering sequence $\mho=\left\{n_{i} \mid i=0,1,2, \ldots\right\}$. From the lemma follows that $W$ is a weakly wandering sequence of bounded type for the transformation $T$.

Let $S$ be a measurable transformation which commutes with the ergodic measure preserving transformation $T$ defined on the infinite measure space $(X, \mathscr{B}, m)$. Since $T$ is ergodic it follows that there exists a constant $K>0$ such that $m(S A)=K m(A)$ for all $A \in \mathscr{B}$. If $S$ does not preserve the measure $m$ then, by possibly interchanging $S$ with its inverse, we may assume that the constant $K$ is greater than 1 . It is also clear that if $B$ is a weakly wandering set under the sequence $W=\left\{n_{i} \mid i=0,1,2, \ldots\right\}$ for the transformation $T$ then the set $S B$ is also a weakly wandering set under the same sequence $\mho$ for $T$. Combining these observations we conclude that $m(S B)>m(B)$. This is a contradiction to the lemma and completes the proof of the theorem. 
The example constructed in [11] possesses such special weakly wandering sequences as described in Theorem 3. However, this fact is not shared by all ergodic measure preserving transformations defined on an infiniite measure space. In [7] examples of ergodic measure preserving transformations $T$ are constructed on the infinite measure space $(X, \mathscr{B}, m)$ of the real line which are unlike the transformation constructed in [11]. Namely, the ergodic measure preserving transformations constructed in [7] commute with measurable transformations that do not preserve the same measure $m$. The examples constructed in [7] seem to have a number of other interesting implications; see [8] and [15]. However, an immediate consequence of the above discussion is that the exhaustive weakly wandering sequences for the transformations constructed in [7] must be of infinite type.

It is possible to make a few statements that relate the weakly wandering sequences of a transformation $T$ and the full group [T] of $T$. However, these are only fragmentary observations at best; and at present, without relying heavily on the known results from the literature, we are unable to give direct and simple proofs of such statements. Nevertheless, there seems to exist helpful information in the structure of the weakly wandering sequences of a transformation $T$ which ought to describe whether $T$ admits a $\sigma$-finite invariant measure $\mu$ equivalent to $m$ or not. We present a sample of such results in the following Theorem 4 which contains no new information, but is merely a rephrasing of known facts. The full group [T] of a transformation $T$ can be regarded as a generalization of the cyclic group generated by $T$. Namely, the full group [T] of a transformation $T$ defined on the measure space $(X, \mathscr{B}, m)$ consists of the set of all measurable transformations $S$ defined on $(X, \mathscr{B}, m)$ such that for all $x \in X, S x=T^{n} x$ for some integer $n=n(x)$.

THEOREM 4. Let $T$ be an ergodic transformation defined on the measure space $(X, \mathscr{B}, m)$, and let $[T]$ be the full group of $T$. Then

(a) If $T$ does not accept any weakly wandering sequences then any transformation $S \in[T]$ does not accept weakly wandering sequences either.

(b) If $T$ accepts an infinite invariant measure $\mu$ equivalent to $m$ then any ergodic transformation $S \in[T]$ accepts weakly wandering sequences.

(c) Let $T$ accept weakly wandering sequences, and suppose there exists an ergodic transformation $S \in[T]$ which does not possess weakly wandering sequences. Then there exists an ergodic transformation $T^{\prime} \in[T]$ such that any ergodic transformation $S^{\prime} \in\left[T^{\prime}\right]$ accepts weakly wandering sequences.

Proor. We would like to mention once more that the above statements are no more than disguised forms of other known results. For that reason we shall only mention the results from the literature that translate to these statements; we leave it to the interested reader to complete the details.

Parts (a) and (b) follow from Theorem 1 of [10]; for part (b) it helps to notice that $S$ is an ergodic transformation preserving the same measure $\mu$ that $T$ preserves. Part (c) is another way of stating part of the Corollary to Theorem 1 of [8].

It is possible that a direct proof of statements of the above nature may 
contain some new and useful ideas; however, we leave such a discussion for another time.

\section{REFERENCES}

1. M. H. Ellis and N. A. Friedman, On eventually weakly wandering sequences (to appear).

2. __ Gap sequences and eventually weakly wandering sequences (to appear).

3. A. Hajian, Strongly recurrent transformations, Pacific J. Math. 14 (1964), 517-523.

4. On ergodic transformations defined on an infinite measure space, Proc. Amer. Soc 16 (1965), 45-48.

5. A. Hajian and Y. Ito, Iterates of measurable transformations and Markov operators, Trans. Amer. Math. Soc. 17 (1965), 371-386.

6. ,Weakly wandering and related sequences, $\mathrm{Z}$. Wahrscheinlichkeitstheorie und Verw. Gebiete 8 (1967), 315-324.

7. A. Hajian, Y. Ito and S. Kakutani, Invariant measures and orbits of dissipative transformations, Advances in Math. 9 (1972), 52-65.

8. , Orbits, sections and induced transformations, Israel J. Math. 18 (1974), 97-115.

9. __ Full groups and a theorem of Dye, Advances in Math. 17 (1975), 48-59.

10. A. Hajian and S. Kakutani, Weakly wandering sets and invariant measures, Trans. Amer. Math. Soc. 110 (1964), 136-151.

11. An example of an ergodic m.p.t. defined on an infinite measure space, Proc. on Ergodic Theory, Lecture Notes in Math., vol. 160, Springer-Verlag, Berlin and New York, 1970.

12. T. Hamachi and M. Osikawa, On zero type and positive type transformations with infinite invariant measure, Mem. Fac. Sci. Kyushu University 25 (1971), 280-295.

13. E. Hopf, Theory of measures and invariant integrals, Trans. Amer. Math. Soc. 34 (1932), 373-393.

14. S. Kakutani, Classification of ergodic groups of automorphisms, Proc. on Func. Anal. and Related Topics, Tokyo, April 1969, pp. 392-397.

15. A problem in equidistribution, Conference in Measure Theory, Oberwolfach 1975, Lecture Notes in Math., vol. 541, Springer-Verlag, Berlin and New York, 1975.

16. U. Krengel and L. Jones, Transformations without a finite invariant measure, Advances in Math. 12 (1974), 275-295.

17. U. Krengel and L. Sucheston, On mixing in infinite measure spaces, Z. Wahrscheinlichkeitstheorie und Verw. Gebiete 13 (1969), 150-164.

Department of Mathematics, Northeastern University, Boston, MassachusetTs 02115

Department of Mathematics, Brown University, Providence, Rhode Island 02912 